\title{
Using Biochemistry to Educate Students on the Causal Link between Social Epigenetics and Health Disparities
}

\author{
Edgar S. Diaz-Cruz, PhD; Michael D. Harland, PharmD; Caleb Darensbourg, PharmD
}

Belmont University College of Pharmacy

\begin{abstract}
Background: While pharmacy education standards require students to recognize social determinants of health (SDOH), there is an opportunity to improve how this is taught in the curriculum. One innovative approach is to educate student pharmacists in a biochemistry course through the integration of topics like epigenetics using SDOH as the framework.

Innovation: A 50-minute educational activity was used to supplement material on the regulation of gene expression, in which epigenetic changes are driven by SDOH. It provided students with a biochemical basis to explain some health disparities, rather than viewing them exclusively as social obstacles to health. The activity employed a mini-lecture, a short video, as well as both small and large group discussion. A reflective paper was used to assess students' understanding of the topic, and the role of the pharmacist in helping patients prevent diseases caused by epigenetic changes due to social determinants of health.

Findings: A post-activity survey showed that the activity increased students' perception of knowledge about SDOH, as well as the effect of epigenetic changes on health outcomes. Furthermore, this activity increased students' awareness about the role that SDOH play in epigenetic changes and challenged students to understand the role that society plays in health outcomes.

Conclusions: The preventable nature of health inequities creates an opportunity to integrate public health into pharmacy education. The integration of epigenetics and SDOH gives the student an opportunity to provide a mechanistic link between social inequities and biochemical processes.
\end{abstract}

Keywords: epigenetics; biochemistry; social determinants of health; health disparities

\section{DESCRIPTION OF THE PROBLEM}

The population of the United States is culturally and ethnically diverse. Diversity, though part of the fabric of this country, may lead to the existence of health disparities. ${ }^{1}$ According to Healthy People 2020, a health disparity is defined as a difference in health that is linked to social, economic, and/or environmental disadvantage, adversely impacting groups that have greater obstacles because of their race or ethnicity. ${ }^{2}$ These social, economic, and environmental factors that impact health disparities include where people are born, work, or live. These factors are termed as social determinants of health (SDOH).

While health inequalities refer to general differences of health between population groups, health inequities are a type of preventable health inequalities that are the result of unjust social policies and practices. This problem of health inequity and the impact of social circumstances on our health will continue to necessitate awareness from health professionals. To address this issue, there have been recent changes in the development and education of future health professionals. ${ }^{3}$ The Accreditation Council for Pharmacy Education (ACPE) established standards to help address this gap in the education of pharmacy professionals. The standard requires students to be able to recognize SDOH using cultural sensitivity as a means to address health disparities. ${ }^{4}$

Corresponding Author: Edgar S. Diaz-Cruz, PhD

Belmont University College of Pharmacy

1900 Belmont Boulevard, Nashville TN 37212-3757

Phone: 615-460-6532; Fax: 615-460-6537

Email: edgar.diaz-cruz@belmont.edu
Even with this change, there remains a disconnect between the proficiency training of cultural sensitivity, and the action of addressing the issues of health disparities. ${ }^{5,6}$ There is a need to continue to refine not only content, but also delivery. As the topic of $\mathrm{SDOH}$ and its impact on health disparities are continually addressed, efforts to employ new integrated curricula in different areas are needed. These issues are not solely a social or public health issue, but have implications in basic sciences, such as biochemistry, that need to be explored with health professionals as a means of education. Within the biochemical sciences, the study of epigenetics, or the modification of DNA expression to induce heritable traits, has been studied in the context of SDOH. This crossroads at the intersection of the social and genetic sciences has developed into the new field of social epigenetics. ${ }^{7,8}$

One innovative approach is to integrate the concepts of social epigenetics into the education of student pharmacists in a traditional lecture-based biochemistry course, using SDOH as a framework for the discussion. The authors are unaware of an existing curricular model for educating healthcare professionals that explains biochemistry concepts through a social epigenetic lens. This integration of the social sciences, molecular biochemistry, and pharmaceutical sciences provides a novel framework for future pharmacy education.

\section{THE INNOVATION}

Epigenetic mechanisms are traditionally covered in biochemistry textbooks as special features involved in the regulation of eukaryotic gene transcription. The classic example used in medical education textbooks involves the 
effect of epigenetic mechanisms in cancer, as it allows instructors to discuss the use of DNA methyltransferase inhibitors (e.g. azacitidine) and histone deacetylase inhibitors (e.g. vorinostat) as potential antineoplastic agents. The idea of developing cancer by altering gene expression through mechanisms that do not involve changes to the underlying DNA sequence is often fascinating to students. However, conventional biochemistry education does very little to explain the process of epigenetic changes, the role society plays in this process or how epigenetic changes contribute to health disparities.

\section{Overview of the Approach}

In this educational activity, SDOH was used as the framework to educate student pharmacists on epigenetic mechanisms. This approach offered students an opportunity to learn about the interplay among SDOH, epigenetics, and health disparities. The objectives of the educational activity were to: 1) define epigenetics, SDOH, and health disparities, 2) discuss the impact of social determinants on health, 3) assess the impact of epigenetic changes on gene expression, and 4) evaluate the role pharmacists play in helping patients prevent diseases caused by epigenetic changes due to SDOH. The activity was conducted over a 50-minute class period and was used to supplement a previous lecture on the regulation of gene expression in which epigenetic changes were first introduced. The approximate allocation of time was: think-pair-share exercise (5 minutes), instructor-led mini lecture including a 5-minute video-clip (20 minutes), large-group discussion and session debrief (20 minutes), and survey (5 minutes).

\section{Educational Activities}

First year student pharmacists registered for a biochemistry course at Belmont University College of Pharmacy over the course of three separate years were included in the evaluation of this educational session ( $n=238)$. The educational activity started with a think-pair-share exercise to have students discuss the following: 1) Are epigenetic changes reversible? 2) Could epigenetic changes alter gene expression patterns permanently? and 3) Explain how epigenetic changes may influence gene expression. A mini lecture followed consisting of background information and examples of epigenetic changes (focusing on DNA methylation), a definition and examples of $\mathrm{SDOH}$, and research data from selected recent studies that show examples of the effect of epigenetic changes on health outcomes linked to SDOH. The video-clip titled: "Epigenetics: Nature vs Nurture" ${ }^{\prime \prime}$ was used to supplement an assigned course reading to discuss the rat study conducted by Weaver et al. in which the investigators measure the effect of maternal stress on offspring epigenetic changes. ${ }^{10}$ The importance of this study lies in the fact that it challenged the notion of health outcomes merely driven by human genetics, but instead considers the effect of social epigenetics as well. A discussion on epigenetic inheritance as the biological basis of $\mathrm{SDOH}$ followed to discuss the effect of epigenetic changes through generations (e.g. mother, fetus, reproductive cells).
The large group discussion started with the notion that epigenetics reveals how the choices patients make can change their genes and those of their kids. The discussion challenged students to reflect on the idea of "choice" versus "fate" and its implications on $\mathrm{SDOH}$. Student pharmacists were asked to reflect on the examples discussed in class about cardiovascular disease in racial/ethnic minorities, breast cancer in black women, head and neck cancer in Blacks and Latinos, obesity, and pre-mature aging. A specific example used in class of the interaction between epigenetics and SDOH involves the DNA methylation status of the $\mathrm{CDH} 13$ promoter. $\mathrm{CDH} 13$ (cadherin 13 ) is an important tumor suppressor in breast cancer patients that has been shown to exhibit significantly differential methylation status in African American women compared to white American women. These altered epigenetic events relevant to racial disparity consequently result in higher rates of cancer development, poor outcome and worse overall survival (Figure 1). ${ }^{11}$ This example highlights a combination of conventional epigenetics in conjunction with a social epigenetics approach. The potential educational outcome of this innovative approach is to allow students to not only recognize the role of epigenetics in cancer development, but also to identify the impact of social determinants on cancer prognosis and outcomes. Moreover, students were asked to reflect on $\mathrm{SDOH}$ that have the potential to negatively impact individuals. Specifically, they looked at socioeconomic status, neighborhood, physical environment, education, access to healthy food, access to health care, as well as community and social context.

At the end of the session, students were asked to complete an anonymous post-activity survey, including basic demographics, using Qualtrics ${ }^{\circledR}$ (Qualtrics Labs Inc., Provo, UT). The survey asked questions regarding perception of knowledge and awareness of the causal link between social epigenetics and health disparities, in addition to assessing their satisfaction with the activity. The survey received an exemption from the Institutional Review Board.

A reflective paper was used to assess students' understanding of the topic. Students were allowed 24 hours to answer the following question: "As a future pharmacist, what do you foresee is your role in helping patients prevent diseases that are caused by epigenetic changes due to SDOH?" The paper was graded based on a rubric for: content and focus (focuses reflection on thoughts and conclusions based on course concepts [20\%]), depth of reflection (demonstrates thorough understanding of concepts and theories discussed in class [30\%]), and analysis (identifies specific examples applicable to pharmacy practice [50\%]). The grading rubric was given to the students as part of the assignment directions. The reflection contributed to $25 \%$ of the in-class activities grade. There were four in-class activities in the course, which collectively contributed to $6.25 \%$ of the final course grade. 


\section{FINDINGS AND CRITICAL ANALYSIS}

A total of 238 students completed the survey for a participation rate of $100 \%$. Students were asked to rate their knowledge about $\mathrm{SDOH}$ before and after completing the educational activity using a 5-point Likert scale (1=strongly disagree and $5=$ strongly agree). Student self-rated knowledge statistically increased after completion of the activity $(2.86 \pm 1.04$ vs. 4.36 $\pm 0.58 ; p<0.0001$ by $t$-test). Furthermore, students were asked to rate their knowledge about the effect of epigenetic changes on health outcomes using the same 5-point Likert scale. Student self-rated knowledge statistically increased after completion of the activity $(2.44 \pm 1.11$ vs. $4.22 \pm 0.65 ; p<0.0001$ by $t$-test).

The discussion and debrief session allowed for constructive dialogue about current challenges faced by pharmacists as they try to serve patients who belong to populations affected by health inequities. The discussion was mainly driven by students' life experiences and interests. While the discussion with students has varied to some extent over the past three years, the discussion often revolves around rural versus urban, racial/ethnic identities, socioeconomic status, immigration status, and sexual orientation-related health disparities.

Students' evaluation of the educational activity was positive (Table 1). A qualitative approach was used to evaluate the reflection papers. Themes related to the pharmacist's role in helping patients prevent diseases caused by epigenetic changes due to SDOH were identified and their frequency was determined. Students described their role as future pharmacists using one or more of these four thematic categories: a) education/counseling (82.0\%), b) advocacy $(37.3 \%)$, c) self-awareness/adaptability (36.1\%), and d) accessibility (9.6\%). Most papers explored specific interventions with a particular patient population that could be spearheaded by pharmacists practicing in a community setting. The average score over three separate years of instruction was $8.9 / 10$ with a range of scores between 6 to 10 . Deductions were made because of a lack of depth of reflection $127.3 \%$ of the papers failing to demonstrate full understanding of the main concepts and $18.2 \%$ of the papers failing to use learned theory to justify their statements) and analysis $(29.5 \%$ of students failing to provide a specific example spearheaded by pharmacists). A key issue in implementation is the training and/or confidence for biochemistry instructor to address SDOH and health disparities. This can be overcome by having a faculty member from the social sciences co-facilitate the activity. This type of approach to teaching epigenetics requires full commitment from the biochemistry instructor and the pharmacy program to provide resources for faculty development in the area of social epigenetics. Furthermore, there is the possibility that some students may be triggered by the comments and/or topics being discussed, due to the nature of health inequities. Similarly, students might feel as if this is a one-time isolated course event. It is important for the instructor to include others involved in delivery of related content to ensure students' knowledge is reinforced in the curriculum during subsequent years of training.

While this activity proved to be effective in educating student pharmacists on SDOH and biochemical concepts, there needs to be a continued effort of integrating this material into the pharmacy curriculum as a whole. Continued blending of concepts like SDOH and genetics can be applied to other topic areas in biochemistry, such as metabolic changes and certain disease states. Building a multifaceted course curriculum is challenging, yet provides an opportunity to teach different topics through varying viewpoints, which leads to a more complex and comprehensive understanding of foundational knowledge. Expanding upon this single activity through future classroom discussions in the social sciences (healthcare delivery, communications, and ethics), as well as in the clinical sciences (therapeutics and case studies) of pharmacy would be beneficial.

\section{CONCLUSIONS}

While the effects of social factors continue to be a focal point when educating student pharmacists on health disparities, an emphasis on the biochemical determinants of cause and health outcomes lags behind. As the role of pharmacy continues to expand, including helping patients with health promotion and disease prevention, so does the role of pharmacy educators to find more innovative teaching approaches that address SDOH. ${ }^{5}$

The preventable nature of health inequities creates an opportunity to integrate public health into pharmacy education. There is a call to action to increase the level of pharmacist involvement in public health efforts. ${ }^{5}$ As a result, it is imperative that programs find innovative ways to include these topics in pharmacy curricula. The integration of epigenetics and SDOH gives the student an opportunity to critically recognize how social determinants disproportionately influence health outcomes. ${ }^{11}$ Furthermore, it encourages students to identify optimal interventions tailored to specific patient populations through counseling and advocacy (Figure 1). The theoretical underpinning to this public health educational approach is to explore how social epigenetics might reveal the biochemical mechanisms underlying health disparities. ${ }^{12}$ The benefit of this teaching approach is that students are able to provide a mechanistic link between social inequities and epigenetic biochemical processes. It allows students to focus on equitable health for all patients rather than focusing on a one-size-fits-all approach to disease prevention and treatment.

Just as the healthcare landscape is evolving and adapting continually, so must the ways in which we present topics to students. With the increasing amount of information new pharmacy professionals must show proficiency in upon graduation, there must be innovative ways of education that layer various topics to enhance learning. As demonstrated through positive evaluations, this educational activity proved to 
be a viable approach for achieving this goal. Notably, this activity offers strategies and resources that can be used and seamlessly implemented by other programs.

Funding/Support: None

Conflicts of Interest: None

\section{REFERENCES}

1. Penman-Aguilar A, Bouye K, Liburd LC, Satterfield D, DeBruyn L, Santos M, et al. Strategies for reducing health disparities - selected CDC-sponsored interventions, United States, 2016. MMWR Suppl 2016;65(No. Suppl 1). https://www.cdc.gov/mmwr/volumes/65/su/pdfs/su 6501.pdf. Accessed 18 August 2020.

2. US Department of Health and Human Services. The Secretary's Advisory Committee on National Health Promotion and Disease Prevention Objectives for 2020. Phase I report: recommendations for the framework and format of healthy people 2020 . Section IV. Advisory Committee findings and recommendations. https://www.healthypeople.gov/sites/default/files/P hasel_0.pdf. Accessed 18 August 2020.

3. Okoro O, Odedina F, Smith WT. Determining the sufficiency of cultural competence instruction in pharmacy school curriculum. Am J Pharm Educ. 2015;79(4):Article 50. doi: 10.5688/ajpe79450

4. Accreditation Council for Pharmacy Education. Accreditation standards and key elements for the professional program in pharmacy leading to the doctor of pharmacy degree (Standards 2016). Approved January 25, 2015. https://www.acpeaccredit.org/pdf/Standards2016FINAL.pdf. Accessed 18 August 2020.
5. Diaz-Cruz ES. If cultural sensitivity is not enough to reduce health disparities, what will pharmacy education do next? Curr Pharm Teach Learn. 2019;11(5):538-540. doi: 10.1016/j.cptl.2019.02.003

6. Okoro ON, Odedina FT, Reams RR, Smith WT. Clinical cultural competency and knowledge of health disparities among pharmacy students. Am J Pharm Educ. 2012;76(3):Article 40. doi: 10.5688/ajpe76340

7. Mansfield B, Guthman J. Epigenetic life: biological plasticity, abnormality, and new configurations of race and reproduction. Cult Geogr. 2015;22(1):3-20. doi: $10.1177 / 1474474014555659$

8. Allis CD, Jenuwein $T$. The molecular hallmarks of epigenetic control. Nat Rev Genet. 2016;17(8):487500. doi: $10.1038 / \mathrm{nrg} .2016 .59$

9. Epigenetics: Nature vs nurture. [Video]. YouTube. https://www.youtube.com/watch?v=k50yMwEOWG U. Published January 29, 2016. Accessed 18 August 2020.

10. Weaver IC, Cervoni N, Champagne FA, et al. Epigenetic programming by maternal behavior. Nat Neurosci. 2004;7(8):847-854. doi: 10.1038/nn1276

11. Ahmad A, Azim S, Zubair H, et al. Epigenetic basis of cancer health disparities: looking beyond genetic differences. Biochim Biophys Acta Rev Cancer. 2017;1868(1):16-28. doi: 10.1016/j.bbcan.2017.01.001

12. Satcher D, Higginbotham EJ. The public health approach to eliminating disparities in health. Am. J. Public Health. 2008;98(3):400-403. doi: 10.2105/AJPH.98.Supplement_1.S8. 
Figure 1. Specific example on how this innovative approach is used to educate students on the interaction between epigenetics and SDOH . ${ }^{11}$

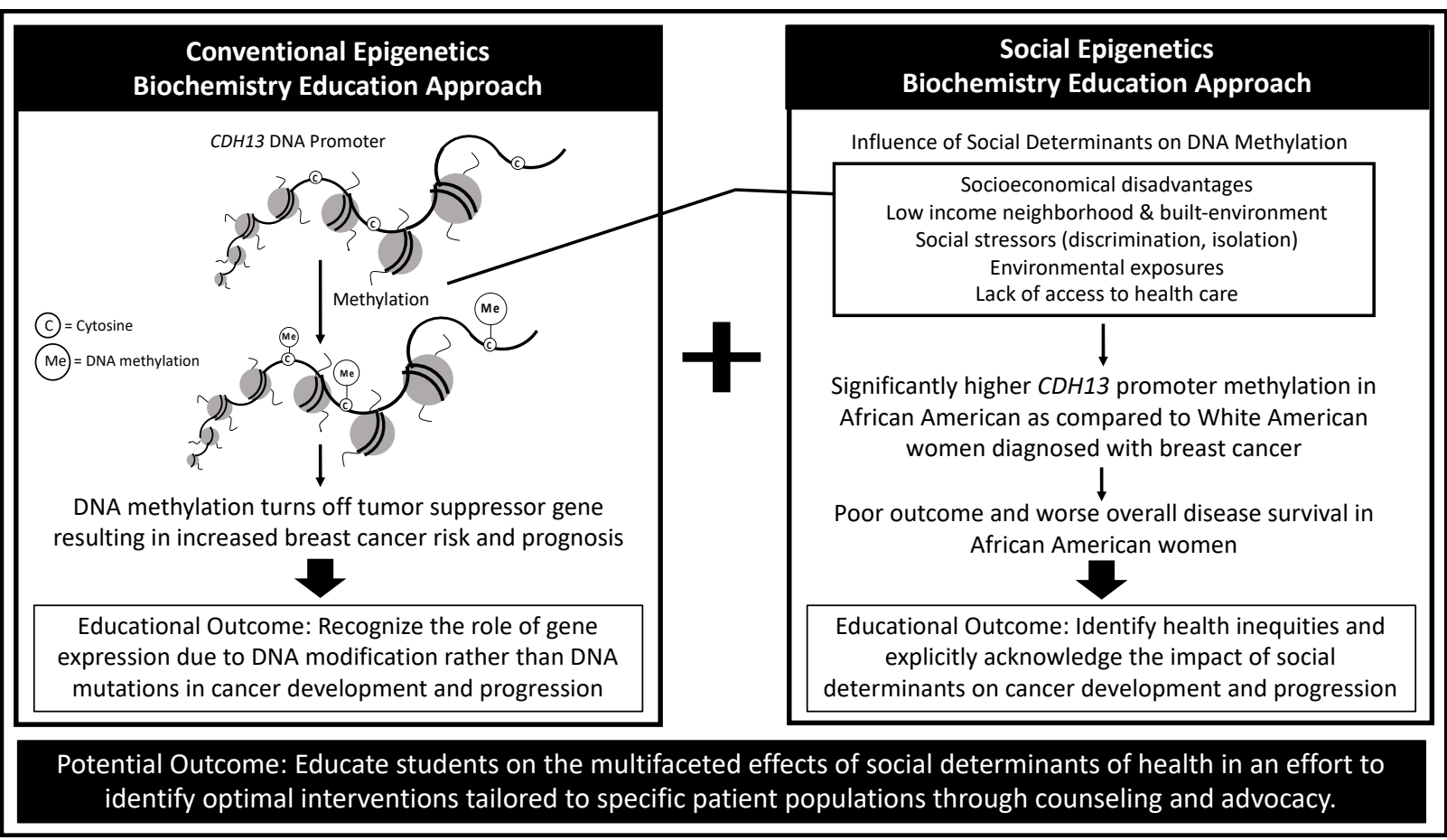

Table 1. Students' evaluation of the education activity

\begin{tabular}{lc}
\hline Statement & Mean \pm SD \\
\hline The activity made me more aware of the role that social determinants of health play on & $4.58 \pm 0.54$ \\
epigenetic changes & $4.41 \pm 0.70$ \\
The activity made me look at biochemistry from a different perspective & $4.49 \pm 0.68$ \\
The activity made biochemistry more relevant to my future profession. & $4.52 \pm 0.66$ \\
The activity helped me see the connection between biochemistry and the patient care & $4.58 \pm 0.58$ \\
process. & $4.64 \pm 0.58$ \\
The activity challenged me to understand the role that society plays in health outcomes. & \\
I would recommend this in-class activity to other student pharmacists.
\end{tabular}

Rating scale: 1 = strongly disagree, 2 = disagree, 3 = neither agree nor disagree, $4=$ agree, $5=$ strongly agree SD - standard deviation 